\title{
Persistence-Length Renormalization of Polymers in a Crowded Environment of Hard Disks
}

\author{
S. Schöbl, S. Sturm, W. Janke, ${ }^{*}$ and K. Kroy ${ }^{\dagger}$ \\ Universität Leipzig, Institut für Theoretische Physik, Postfach 100 920, D-04009 Leipzig, Germany
}

(Dated: October 23, 2014)

\begin{abstract}
The most conspicuous property of a semiflexible polymer is its persistence length, defined as the decay length of tangent correlations along its contour. Using an efficient stochastic growth algorithm to sample polymers embedded in a quenched two-dimensional hard-disk fluid, we find apparent wormlike chain statistics with a renormalized persistence length. We identify a universal form of the disorder renormalization that suggests itself as a quantitative measure of molecular crowding.
\end{abstract}

PACS numbers: 87.15.A-, 87.15.ak, 87.16.Ln

Single-molecule experiments have established the wormlike chain (WLC) as a standard model for semiflexible biopolymers [1-5]. It emerges from the classical Heisenberg model for a ferromagnetic spin chain in the continuum limit, where the spins merge into an inextensible space curve $\mathbf{r}_{s}$ and the exchange interaction between adjacent spins (or unit tangent vectors $\mathbf{r}_{s}^{\prime}$ ) turns into an energetic penalty for bending. The resulting continuum Hamiltonian $\frac{\kappa}{2} \int\left(\mathbf{r}_{s}^{\prime \prime}\right)^{2} \mathrm{~d} s$ is also familiar from continuum mechanics as a model for a slender rod with bending rigidity $\kappa[6]$. The WLC model accurately describes a very diverse range of polymers, including DNA [1], muscle protein [2], filamentous actin [3], as well as synthetic carbon nanotubes $[7,8]$.

Exploiting the analogy of the WLC with the Heisenberg magnet, it can be shown [9] that for an isolated WLC in $d$ dimensions, the equilibrium tangent-tangent correlation function decays exponentially,

$$
\left\langle\mathbf{r}_{s}^{\prime} \cdot \mathbf{r}_{s+\Delta s}^{\prime}\right\rangle=\exp \left(-|\Delta s| / \ell_{p}\right),
$$

and thus defines a thermal persistence length

$$
\ell_{p}=\frac{2 \kappa}{(d-1) k_{B} T} .
$$

The analytically solvable case of a single polymer in isolation maps well to single-molecule experiments but bears little resemblance to the disordered environment provided by a surrounding polymer network or even the cytoplasm [10-12]. Although much work has been done on both local [13-17] and global [18, 19] properties of semiflexible polymers in disorder, it remains unknown just how much of the WLC survives in the presence of a disordered environment. The exponentially decaying tangent-tangent correlation function in Eq. (1) might for example turn into some nonexponential function of $\Delta s$, or it might remain exponential and thus define a renormalized persistence length, which then may or may not agree with its thermal value, given by Eq. (2). In the last case, one can imagine that the persistence length increases (due to channel formation [20]) or decreases (due to crumpling induced by the obstacles) with respect to its thermal value.
For an isolated WLC, the exponential decay described by Eq. (1) derives from a random walk in tangent space, which is most easily visualized by imagining a thermal ensemble of polymers with one of their ends held clamped along a given direction. Their free ends will then point along the same direction if the polymers are very short, but stray away from it diffusively if the polymers are longer. Conversely, if the same ensemble is exposed to a quenched random background that is in some regions more favorable than in others, the free polymer ends will naturally gravitate towards the more favorable regions. It has long been established that this can give rise to a superdiffusive growth of transverse fluctuations for directed polymers in random media $[21,22]$. Recent results by Boltz and Kierfeld show that it can also give rise to a superdiffusive growth of tangent fluctuations for stiff semiflexible polymers exposed to $\delta$-correlated quenched Gaussian disorder [23]. A superdiffusive growth of tangent fluctuations translates to a faster decay of tangent-tangent correlations compared to an isolated WLC, which on a scaling level can be captured by an "effective disorderinduced persistence length" [23] that is smaller than the thermal value Eq. (2). It also translates, however, to a tangent-tangent correlation function decaying faster than exponentially, and thus does not define a persistence length in the strict sense of Eq. (1).

To find out whether a renormalized persistence length in the strong sense of Eq. (1) might be observed under more realistic conditions, we have performed extensive numerical simulations of two-dimensional semiflexible polymers in a quenched equilibrium hard-disk fluid. As we demonstrate below, this disorder-averaged ensemble of test polymers indeed exhibits a renormalized persistence length in the strong sense, which can account also for the dominant effect of the crowding onto the polymer end-to-end distribution. This suggests that even if a shape analysis of microscopic in vivo imagery seems to agree perfectly well with standard WLC results, the persistence length inferred from the data may deviate significantly from the polymer's thermal persistence length that obeys Eq. (2). For sufficiently stiff polymers, we find that the renormalized persistence length can be de- 
termined uniquely from the thermal persistence length and an auxiliary quantity that characterizes the disordered environment. This feature, which is known to hold true in the double asymptotic limit of high stiffness and $\delta$-correlated Gaussian disorder [23, 24], but to our knowledge never has been observed in a more realistic setting, opens a novel way to employ polymers of known stiffness as quantitative probes of molecular crowding.

Our simulations are based on the discrete representation of the polymer, which is constrained to lie in a plane $(d=2)$. The discrete (Heisenberg) and continuum (WLC) forms of the Hamiltonian read

$$
\mathcal{H}=-\frac{\kappa}{b} \sum_{i=1}^{N-1} \mathbf{t}_{i} \cdot \mathbf{t}_{i+1} \simeq \frac{\kappa}{2} \int_{0}^{L}\left(\theta_{s}^{\prime}\right)^{2} \mathrm{~d} s .
$$

The unit tangent $\mathbf{r}_{s}^{\prime}$ at arclength position $s=i b$, corresponding to the $i^{\prime}$ th spin $\mathbf{t}_{i} \equiv\left(\mathbf{r}_{i+1}-\mathbf{r}_{i}\right) / b$, has been identified with its angle $\theta_{s}$ in the plane. It diffuses freely on the unit circle as a function of the arclength, so that the increments $\Delta \theta$ are Gaussian distributed according to

$$
\frac{1}{\sqrt{2 \pi} \sigma} \exp \left[-\frac{(\Delta \theta)^{2}}{2 \sigma^{2}}\right], \quad \sigma^{2} \equiv \frac{k_{B} T}{\kappa} \Delta s .
$$

This induces an exponential decay of tangent correlations, which yields, by comparison with Eq. (1), the thermal persistence length $\ell_{p}=2 \kappa / k_{B} T$. In the following, we subject the polymer to a random, quenched and statistically isotropic background potential $V(\mathbf{r})$. In contrast to earlier analytical works on the subject $[18,19]$ we are free to do away with the simplifying assumptions of a vanishing correlation length or a Gaussian distribution of potential energies; instead we adhere closely to the spirit of molecular crowding by assuming sterically interacting obstacles that are small compared to the polymer, but still large on the monomer scale. As a paradigmatic representative of steric disorder, we consider an equilibrated (but quenched with respect to the polymer) hard-disk fluid at several area filling fractions $\phi$, ranging from $40 \%$ to the verge of the freezing transition at $\phi \approx 70 \%$ [25]. Then $V(\mathbf{r})$ takes the values $\infty$ or 0 , depending on whether the polymer penetrates any of the disks or not. Since in an unbounded quenched system the polymer as a whole will gravitate towards ever more favorable regions [18], thus in our case producing a trivial ensemble of straight rods in the limit of large persistence lengths and infinitely large systems, we eliminate the dependence on system volume by fixing one polymer end at the origin, $\mathbf{r}_{0}=(0,0)$, which one might think of as a membrane-bound anchor point in the context of biopolymers in cells.

The extreme strength and density of environmental interactions present a formidable challenge to conventional Monte Carlo simulation schemes, which we found hard to overcome even using a sophisticated multicanonical histogram reweighting procedure [26]. We have therefore adopted a breadth-first growth algorithm [27] that resolves this difficulty by circumventing energy barriers instead of trying to cross them [20, 26]. For a given disorder realization, the algorithm starts with an ensemble of monomers fixed at the origin and then performs $N-1$ successive growth steps to extend each ensemble member to a polymer of the desired length. During each growth step, every polymer in the ensemble branches out into a small set of new trial configurations through the addition of monomers pointing in random directions. This incurs a severalfold increase of the ensemble population. Next, the Boltzmann weight of each new trial configuration is calculated. Each trial configuration is then either eliminated or replicated probabilistically, such that (i) the overall ensemble population is held approximately constant to prevent memory and processing time requirements from growing exponentially and (ii) the occurrence probability of any given configuration agrees with its equilibrium probability $\propto \exp \left(-\beta \mathcal{H}\left[\left\{\mathbf{r}_{i}\right\}\right]\right)$, thus establishing thermal equilibrium after each growth step.

We analyze our numerical data first in terms of the length-averaged tangent correlation function

$$
\mathcal{C}(\Delta s) \equiv \frac{1}{L-\Delta s} \int_{0}^{L-\Delta s} \overline{\left\langle\mathbf{t}_{s} \cdot \mathbf{t}_{s+\Delta s}\right\rangle} \mathrm{d} s,
$$

where the overbar denotes the additional disorder average. As demonstrated in Fig. 1, $\mathcal{C}(\Delta s)$ still decays exponentially in the mean. Disorder-induced non-exponential modulations are found to decay in amplitude as the thermal persistence length $\ell_{p}=2 \kappa /\left(k_{B} T\right)$ increases compared to the obstacle size $D$ and the polymer length $L$ - or, equivalently, upon decreasing the temperature $T$. Already at modest thermal persistence lengths $\ell_{p} \approx L / 2$, a value easily realizable in experiment with filamentous actin or carbon nanotubes, the deviations from perfect exponentiality nowhere exceed $3 \%$. This behavior, which we would not necessarily have anticipated, justifies the notion of a persistence-length renormalization [28]. Every disorder-averaged polymer ensemble defined by a thermal persistence length and a given density and size of background disks can therefore be characterized by an apparent renormalized persistence length $\ell_{p}^{*}$ inferred from fitting $\exp \left[-\Delta s / \ell_{p}^{*}\right]$ to Eq. (5), as exemplified in Fig. 1.

The fit results are shown in Fig. 2 for two representative disorder filling fractions $\phi$ and thermal persistence lengths $\ell_{p}$ between $2 D$ and $10 D$. The total polymer length $L$ is 10 disk diameters $D$ and the discretization length $b=D / 5$. The inferred values for $\ell_{p}^{*}$ systematically decrease with increasing disorder filling fraction for all but the smallest thermal persistence length $\ell_{p}=2$. While for $\delta$-correlated Gaussian disorder, the renormalized persistence length $\ell_{p}^{*}$ should actually change with $L$ [23], no significant $L$ dependence of $\ell_{p}^{*}$ can be detected within the range of contour lengths accessible in our simulations [29]. As $L$ in our simulations is comparable to the thermal persistence length $\ell_{p}$, and several times larger than the obstacle size, 

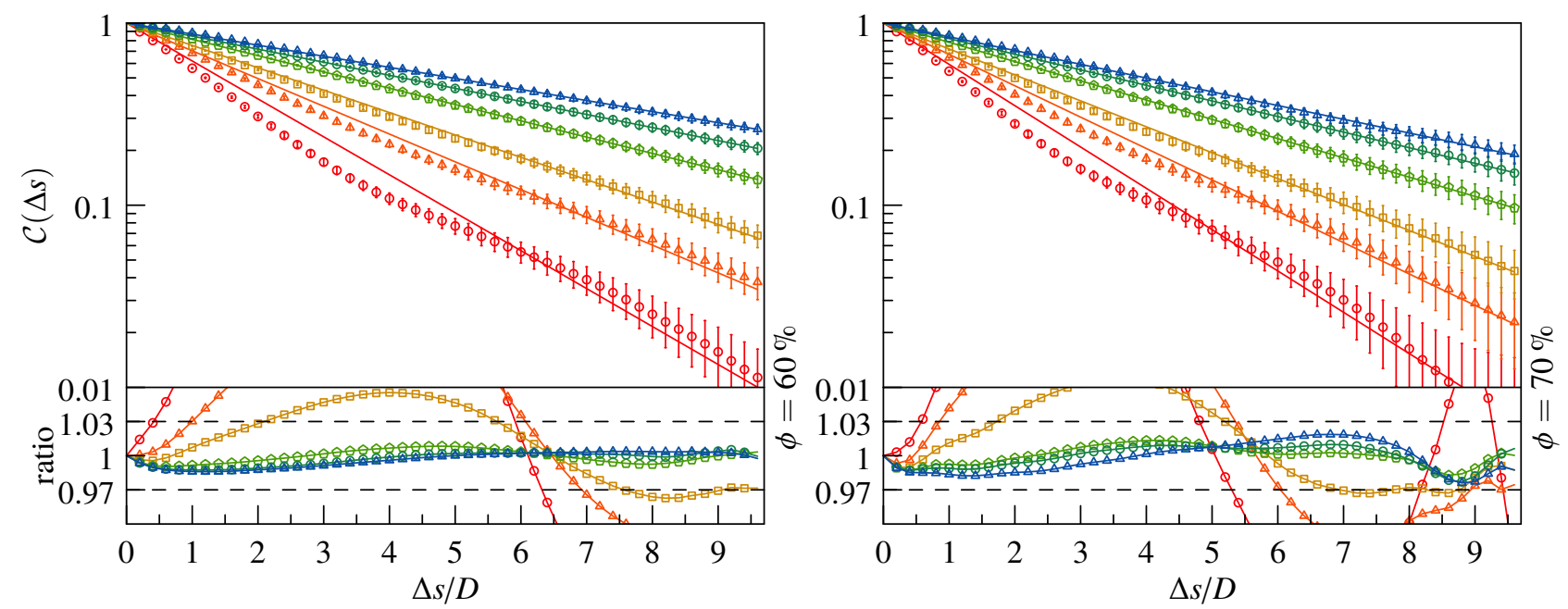

FIG. 1. Numerically obtained disorder-averaged tangent correlation function $\mathcal{C}$ as a function of the reduced backbone distance for background filling fractions $\phi=60 \%, 70 \%$ and thermal persistence lengths $\ell_{p} / D=2,3,4,6,8,10$ (from bottom to top). Solid lines indicate our exponential fits $\mathcal{C}(\Delta s)=\exp \left(-\Delta s / \ell_{p}^{*}\right)$. For stiff polymers $\ell_{p} \gtrsim 5 D$ the relative error of the exponential fit remains bounded to about $3 \%$ (bottom panels).

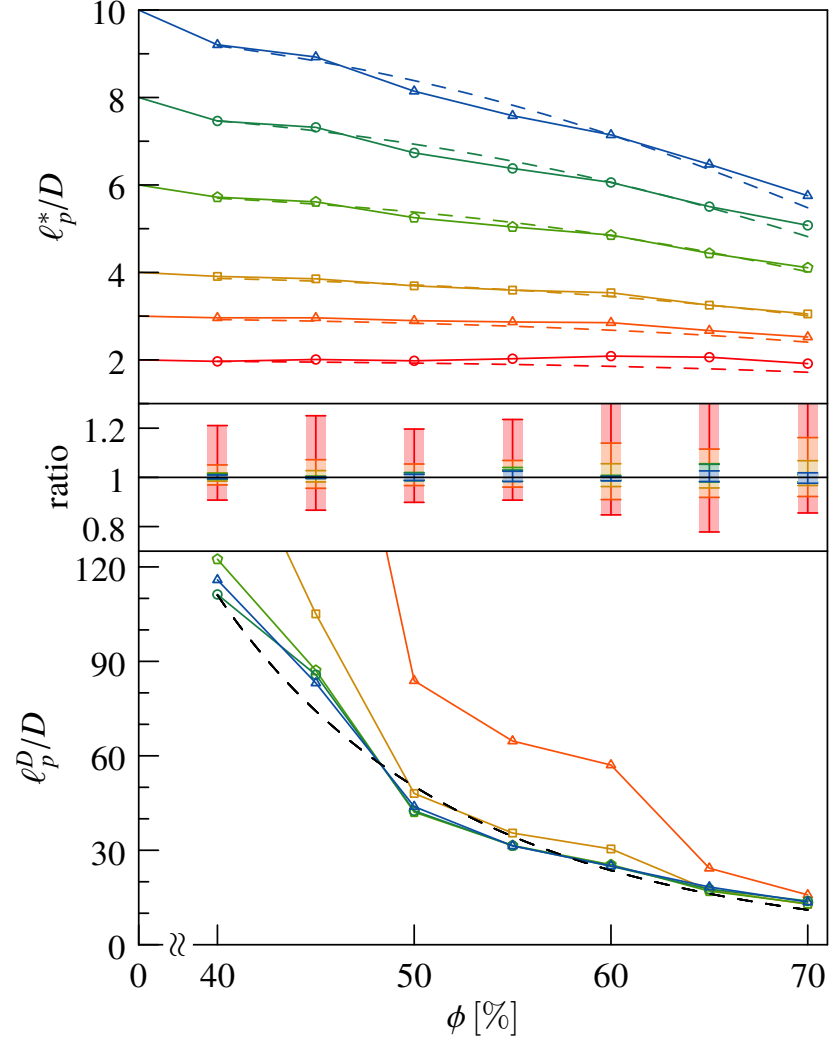

FIG. 2. Renormalized persistence lengths $\ell_{p}^{*}$ resulting from the exponential fits in Fig. 1 for thermal persistence lengths $\ell_{p} / D=2,3,4,6,8,10$ (upper panel/solid lines, from bottom to top) and Eq. (7) (dashed). The maxima of the relative fit errors (lower panel of Fig. 1) are indicated as bars (middle panel). For $\ell_{p}>2 D$ the disorder persistence length $\ell_{p}^{D}=\left(1 / \ell_{p}^{*}-1 / \ell_{p}\right)^{-1}$ (bottom panel) quickly converges onto a single $\ell_{p}$-independent master curve as implied by Eq. (8) (dashed line). these contour lengths should closely approximate typical experimental conditions in cell biophysics.

The observed exponential scaling of tangent-tangent correlations implies that the renormalized persistence length $\ell_{p}^{*}$ still derives from a diffusion process in tangent space (as for a free WLC), albeit with a renormalized diffusivity. It is useful to note at this point that our model system exhibits, in the limit of vanishing obstacle size $D \rightarrow 0$ and diverging persistence length $\ell_{p} \rightarrow \infty$, the so-called tilt symmetry $[23,24]$. It causes the disorder-averaged angular fluctuations to separate into an unperturbed, "thermal" part and a disorder-induced part. Together with the observed $L$-independent persistence length renormalization, we thus find the asymptotic relation

$$
\overline{\left\langle\left(\Delta \theta^{2}\right)\right\rangle} /(2 \Delta s)=1 / \ell_{p}^{*}=1 / \ell_{p}+1 / \ell_{p}^{D},
$$

which defines a "disorder persistence length" $\ell_{p}^{D}\left(\phi, D, \ell_{p}\right)$ that should converge onto a $\ell_{p}$-independent master curve, in the limit $\ell_{p} \rightarrow \infty$. This prediction is in fact well supported by our data, despite the finite size of the obstacles; see Fig. 2 (bottom). As a consequence, we can even rationalize the form of this master curve, on a scaling level. Namely, the pure disorder effect onto the polymer conformation may simply be represented as a succession of $D$-sized deflections, separated by some distance $\ell$ that roughly corresponds to the "mean free path" between subsequent polymer-obstacle collisions. Each real-space deflection of size $D$ amounts to a rotation $\delta \theta=D / \ell$ in tangent space, hence giving rise to a disorder persistence length $\ell_{p}^{D}=2 \ell^{3} / D^{2}$. The mean free path $\ell$ should scale with $\phi^{-1 / 2}$ at small disorder densities and approach a value on the order of $D$ at hexagonal close packing, $\ell\left(\phi_{\mathrm{hcp}} \approx 90.7 \%\right) \approx D$. Indeed we find that with the semi-empirical form $\ell / D \approx 5.3\left(\phi^{-1 / 2}-\phi_{\mathrm{hcp}}^{-1 / 2}\right)+1$ the 


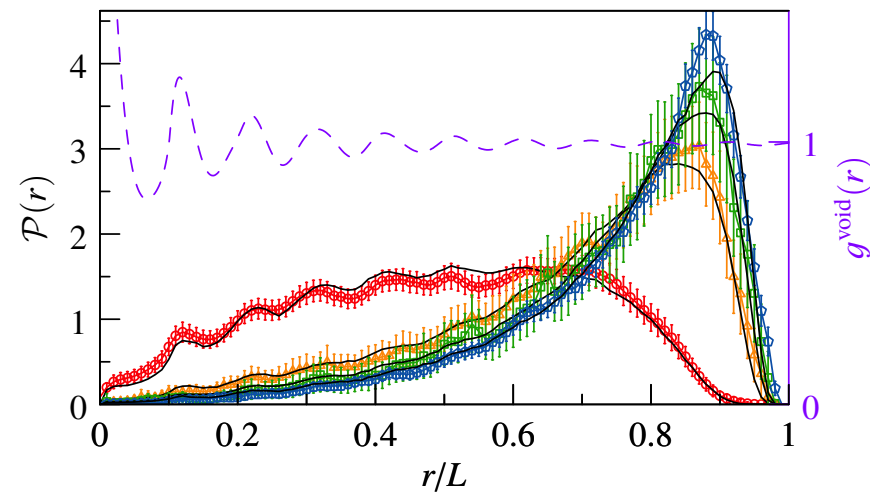

FIG. 3. Numerical disorder-averaged radial distribution functions $\mathcal{P}(r)$ for thermal persistence lengths $\ell_{p} / D=2,6,8,10$ (data) and $\phi=70 \%$. Solid lines represent a parameter-free comparison with free WLC radial distribution functions evaluated for the corresponding renormalized persistence lengths $\ell_{p}^{*}$ obtained from Fig. 2 and multiplied by the normalized void distribution function $g^{\text {void }}(r)$ (dashed).

argument provides an accurate analytical parametrization of the renormalized persistence length $\ell_{p}^{*}\left(\phi, D, \ell_{p}\right)$,

$$
D / \ell_{p}^{*} \approx D / \ell_{p}+\frac{1}{2}\left[5.3\left(\phi^{-1 / 2}-\phi_{\mathrm{hcp}}^{-1 / 2}\right)+1\right]^{-3} .
$$

Figure 2 compares Eq. (7) and its polymer-independent asymptotics for $\ell_{p} \rightarrow \infty$,

$$
\ell_{p}^{*} \sim \ell_{p}^{D}(\phi, D) \approx 2 D\left[5.3\left(\phi^{-1 / 2}-\phi_{\mathrm{hcp}}^{-1 / 2}\right)+1\right]^{3},
$$

to our numerical data. Note that both $\ell_{p}^{*}$ and $\ell_{p}$ can be determined experimentally, so that $\ell_{p}^{D}=\ell_{p}^{*}\left(\ell_{p} \gg D\right)$ provides a practical quantitative measure of the environmental disorder strength. Equation (8) and possible refinements for polydisperse obstacle sizes, might thus prove useful in future attempts to quantify cellular crowding in terms of the density and size (distribution) of the steric obstacles.

Although effective semiflexibility re-emerges on the global level of tangent correlations, more localized observables must bear witness to the presence of disorder correlations, allowing one to distinguish experimentally between "true" and "renormalized" semiflexibility, and providing further information on the nature of the obstacles and their correlations. Here, we discuss the disorder-averaged radial distribution function,

$$
\mathcal{P}(r) \equiv 2 \pi r \overline{\left\langle\delta\left(\mathbf{r}-\mathbf{r}_{L}+\mathbf{r}_{0}\right)\right\rangle}, \quad \text { with } r \equiv|\mathbf{r}|,
$$

as an important example of such more local observables. As seen from Fig. 3, $\mathcal{P}(r)$ picks up the fluid structure of the background, as the ensemble branches out inside the more expansive voids and circumvents denser regions. To a first approximation, $\mathcal{P}(r)$ factorizes into two contributions: the free WLC radial distribution $[30,31]$ with the renormalized persistence length $\ell_{p}^{*}$ and a factor weighing the relative abundance of void space at a given distance $r$. As demonstrated in Fig. 3, the latter is well represented by the "void space distribution function",

$$
g^{\operatorname{void}}(r) \propto r^{-1} \overline{\int \mathrm{d} \mathbf{r}^{\prime} \delta\left(\left|\mathbf{r}^{\prime}\right|-r\right) e^{-\beta V(\mathbf{0})} e^{-\beta V\left(\mathbf{r}^{\prime}\right)}},
$$

a close cousin to the radial distribution function known from liquid-state theory.

Our results suggest that the molecular crowding in the cytoplasm of cells will crumple embedded cytoskeletal polymers. Although we are not the first to predict that a quenched disordered background should induce a renormalized persistence length [23], we were able to show explicitly that tangent correlations remain exponential, even at the highest filling fractions and for finite obstacle size. This indicates that the common practice of performing a static shape analysis of single fluorescently labeled polymers in vivo or in in-vitro reconstituted polymer solutions and networks requires special caution. It may not yield a reliable estimator of intrinsic polymer stiffness, even if its results look deceptively consistent with the WLC model. In spite of the modest size difference between the polymer length and the range of background correlations, we found that, for sufficiently stiff test polymers, the renormalized persistence length is uniquely determined in terms of the thermal persistence length $\ell_{p}$ and a "disorder persistence length" $\ell_{p}^{D}$ that characterizes the ambient disorder. On this basis, polymers of known intrinsic stiffness can be used as generic quantitative probes of molecular crowding. With our simple formula for the renormalized persistence length, their tangent correlations and radial distribution are conveniently analyzed in terms of the background disorder parameters.

It is an intriguing question whether our findings generalize to three dimensions. We assume they do, at least for generic kinds of disorder such as a random distribution of spheres or bent rods: neither the concept of tilt symmetry nor the idea of random polymer-obstacle collisions are specific to two dimensions. Our "disorder persistence length" has a simple definition and could in principle be measured using standard video microscopy techniques analyzed in the usual way (by measuring tangent-tangent correlations and radial distribution functions). Therefore, we hope to inspire not only further numerical or analytical work on the matter, but also experimental studies under physiological conditions. In this context, it would also be interesting to extend our analysis to the case of annealed disorder, which has recently been addressed experimentally for flexible polymers [32].

\section{ACKNOWLEDGEMENTS}

We thank Johannes Zierenberg, Niklas Fricke, Martin Marenz and Jan Kierfeld for fruitful discussions and ben- 
eficial advice and acknowledge financial support from the Leipzig Graduate School of Excellence GSC185 "BuildMoNa", from the German Science Foundation via FOR877 and SFB/TRR 102, the European Union and the Free State of Saxony, and from the Deutsch-Französische Hochschule (DFH-UFA).

* wolfhard.janke@itp.uni-leipzig.de

† klaus.kroy@uni-leipzig.de

[1] C. Bustamante, Z. Bryant, and S. Smith, Nature 421, 423 (2003).

[2] M. Rief, M. Gautel, F. Oesterhelt, J. Fernandez, and H. Gaub, Science 276, 1109 (1997).

[3] F. Gittes, B. Mickey, J. Nettleton, and J. Howard, J. Cell Bio. 120, 923 (1993).

[4] J. F. Marko and E. D. Siggia, Macromolecules 28, 8759 (1995).

[5] O. Otto, S. Sturm, N. Laohakunakorn, U. F. Keyser, and K. Kroy, Nature Comm. 4, 1780 (2013).

[6] L. Landau and E. Lifshitz, Theory of Elasticity (Pergamon, Oxford, 1986).

[7] M. Sano, A. Kamino, J. Okamura, and S. Shinkai, Science 293, 1299 (2001).

[8] A. W. Barnard, V. Sazonova, A. M. van der Zande, and P. L. McEuen, Proc. Nat. Acad. Sci. U.S.A. 109, 19093 (2012).

[9] C. Thompson, Classical Equilibrium Statistical Mechanics (Clarendon, Oxford, 1988).

[10] R. Ellis, Trends Biochem. Sci. 26, 597 (2001).

[11] M. Weiss, M. Elsner, F. Kartberg, and T. Nilsson, Biophys. J. 87, 3518 (2004).

[12] F. Höfling and T. Franosch, Rep. Prog. Phys. 76, 046602 (2013).

[13] H. Hinsch, J. Wilhelm, and E. Frey, Eur. Phys. J. E 24, 35 (2007).

[14] J. Glaser, D. Chakraborty, K. Kroy, I. Lauter, M. Degawa, N. Kirchgeßner, B. Hoffmann, R. Merkel, and M. Giesen, Phys. Rev. Lett. 105, 37801 (2010).

[15] J. Glaser and K. Kroy, Phys. Rev. E 84, 051801 (2011).
[16] D. Sussman and K. Schweizer, Phys. Rev. Lett. 107, 78102 (2011).

[17] H. Hinsch and E. Frey, ChemPhysChem 10, 2891 (2009).

[18] M. Cates and R. Ball, J. Phys. (Paris) 49, 2009 (1988).

[19] A. Dua and T. Vilgis, J. Chem. Phys. 121, 5505 (2004).

[20] S. Schöbl, J. Zierenberg, and W. Janke, J. Phys. A 45, 475002 (2012).

[21] M. Kardar, Statistical Physics of Fields (Cambridge University, Cambridge, 2007).

[22] T. Halpin-Healy and Y. Zhang, Phys. Rep. 254, 215 (1995).

[23] H. Boltz and J. Kierfeld, Phys. Rev. E 88, 012103 (2013).

[24] T. Hwa and D. S. Fisher, Phys. Rev. B 49, 3136 (1994).

[25] E. P. Bernard and W. Krauth, Phys. Rev. Lett. 107, 155704 (2011).

[26] S. Schöbl, J. Zierenberg, and W. Janke, Phys. Rev. E 84, 051805 (2011).

[27] T. Garel and H. Orland, J. Phys. A 23, L621 (1999).

[28] That the effect of crowding on the statistical conformation of the test polymer can essentially be captured by a renormalized persistence length $\ell_{p} \rightarrow \ell_{p}^{*}$ does not imply a renormalized bending rigidity $\kappa \rightarrow \kappa^{*}$, as introduced in a different context [33]; $\kappa$ is a microscopic parameter of the Hamiltonian in Eq. (3), $\ell_{p}$ is a statistical observable defined by Eq. (1).

[29] Note that even within the strong-disorder regime as defined by Boltz and Kierfeld, the predicted deviation from normal tangent diffusion only scales with a small power of $L, \Delta \theta^{2} \sim L^{1.18 \ldots}$ [23]. To clearly distinguish between a true exponential decay of tangent-tangent correlations that extends to $L \rightarrow \infty$, and a slow crossover akin to Eq. (43) in Ref. [23] would require us to further extend $L$ beyond the limits of current numerical methods. This does not diminish the relevance of our results to biophysical experiments, where polymer lengths typically do not vary over several orders of magnitude.

[30] J. Wilhelm and E. Frey, Phys. Rev. Lett. 77, 2581 (1996).

[31] B. Hamprecht, W. Janke, and H. Kleinert, Phys. Lett. A 330, 254 (2004).

[32] A. Soranno, I. Koenig, M. B. Borgia, H. Hofmann, F. Zosel, D. Nettels, and B. Schuler, Proc. Nat. Acad. Sci. U.S.A. 111, 4874 (2014).

[33] P. Gutjahr, R. Lipowsky, and J. Kierfeld, EPL (Europhys. Lett.) 76, 994 (2006). 